\title{
THE N-VORTEX PROBLEM ON A SYMMETRIC ELLIPSOID: A PERTURBATION APPROACH.
}

\author{
CÉSAR CASTILHO $^{\dagger *}$ AND HÉLIO MACHADO ${ }^{\dagger}$ \\ ${ }^{\dagger}$ Departamento de Matemática \\ Universidade Federal de Pernambuco \\ Recife, PE CEP 50740-540 Brazil \\ and \\ * The Abdus Salam ICTP \\ Strada Costiera 11 \\ Trieste 34100 Italy
}

\begin{abstract}
We consider the N-vortex problem on a ellipsoid of revolution. Applying standard techniques of classical perturbation theory we construct a sequence of conformal transformations from the ellipsoid into the complex plane. Using these transformations the equations of motion for the $\mathrm{N}$-vortex problem on the ellipsoid are written as a formal series on the eccentricity of the ellipsoid's generating ellipse. First order equations are obtained explicitly. We show numerically that the truncated first order system for the three-vortices system on the symmetric ellipsoid is non-integrable.
\end{abstract}

Key Words: Vortex Dynamics, Hamiltonian Systems, Perturbation Theory.

MSC: 76C05, 58F40, 70H05.

\section{INTRODUCTION}

The equations of point vortices motion in the plane were introduced by Helmholtz [1] and described as an Hamiltonian system by Kirchhoff in 1876 2]. These equations were first generalized to describe the motion of pointvortices on a sphere by Bogolmonov in 1977 [3. Bogomolnov's work was put into solid mathematical grounds by Kimura and Okamoto in 1987 [5]. Independently Hally wrote in 1979 [4 the equations for vortices motion in symmetric surfaces of revolution (which englobe the sphere). To write his equations Hally represented the surface $M$ on the complex plane with a metric conformal to the Euclidean metric: Let the $i$-th vortex, with vorticity $\Gamma_{i}$ 
be represented by the complex coordinate $\xi_{i}$. Calling the surface's conformal factor $h(\xi, \bar{\xi})$, Hally's equations are

$$
\bar{\xi}_{n}^{\prime}=h^{-2}\left(\xi_{n}, \bar{\xi}_{n}\right)\left(\sum_{k}^{\prime}-i \frac{\Gamma_{k}}{\xi_{n}-\xi_{k}}+i \Gamma_{n} \frac{\partial}{\partial \xi_{n}} \log \left(h\left(\xi_{n}, \bar{\xi}_{n}\right)\right)\right) .
$$

The difficulty in working with these equations is that one must know explicitly the conformal factor $h(\xi, \bar{\xi})$ and this factor is not known for many surfaces but the sphere.

The aim of this paper is to write the equations for the N-vortex problem on the ellipsoid of revolution

$$
\frac{x^{2}}{R^{2}}+\frac{y^{2}}{R^{2}}+\frac{z^{2}}{R^{2}(1+\epsilon)}=1
$$

as an $\epsilon$-series when $|\epsilon|<<1$. To achieve this goal we write a perturbative coordinate transformation from the ellipsoid into the plane (see (16) and impose conformality at each order. It turns out that the transformation's n-th order term is given by the solution of a certain linear differential equation and that the first order term can be computed explicitly (see (3i)). Using Hally's equations we calculated rigorously the first order perturbation term for the vortex equations on the ellipsoid. In spherical coordinates the truncated first order equations are given by the hamiltonian system with hamiltonian

$$
H=H_{0}+\epsilon H_{1}
$$

where

$$
\begin{gathered}
H_{0}=\frac{1}{2} \sum_{i, j}^{\prime} \Gamma_{i} \Gamma_{j}\left[\ln \left(2-2 \cos \left(\theta_{i}\right) \cos \left(\theta_{j}\right)-2 \sin \left(\theta_{i}\right) \sin \left(\theta_{j}\right) \cos \left(\phi_{i}-\phi_{j}\right)\right)\right], \\
H_{1}=\frac{1}{2} \sum_{i, j}^{\prime} \Gamma_{i} \Gamma_{j}\left[\left(\cos \left(\theta_{i}\right)^{2}+\cos \left(\theta_{j}\right)^{2}\right)\right]
\end{gathered}
$$

and symplectic two-form

$$
w=\sum_{i=1}^{N} \Gamma_{i} \sin \left(\theta_{i}\right) d \theta_{i} \wedge d \phi_{i}
$$

The paper is organized as follows: In section (2) we introduce all the concepts used in the derivation of the vortex equations. Hally's equations are written as an invariant hamiltonian system and, for the spherical case, are shown to be equivalent to Bogolmonov's equations. In section (3) we derive the expression for the first order perturbation term. The main idea is to write the conformal transformation from the ellipsoid into the plane as a formal series in the eccentricity of the generating ellipse of the ellipsoid. In section (4) we exhibit two simple applications of equations (2). The case $N=2$, despite being integrable, seems not to admit closed solutions. We show that, when $\left|\Gamma_{1}\right|=\left|\Gamma_{2}\right|$, the only solutions for which the two vortices 
maintain their relative distance constant are the relative equilibria. For the $N=3$ case, we show numerically, by choosing specific parameters, that the three-vortices problem (2) on the ellipsoid is chaotic.

\section{The Equations of Hally}

In this section we discuss equations (11) from a geometric and invariant point of view. Hally's equations were deduced under the topological constraint that the sum of all vorticities is zero and, in fact, this is essential in recasting the equations in Hamiltonian form. We also show, that, under this zero vorticity constraint, Hally's equations on the sphere, coincide with Bogolmonov's equations.

2.1. Hally's equations as an hamiltonian system on the conformal plane. Let $M$ be a 2-dimensional Riemannian manifold with metric tensor g. $M$ is a symplectic manifold with symplectic form $w$ given by the area form induced by $g$. Let $H: M \rightarrow \mathbb{R}$ be the hamiltonian function. Hamilton's equations [9] are

$$
w\left(X_{H}, \cdot\right)=d H
$$

where $X_{H}$ is the hamiltonian vector field induced by $H$. Now we assume that $M$ is conformal to the plane, that is, that there are coordinates $x_{i}: M \rightarrow$ $\mathbb{R}^{2} ; i=1,2$ where the metric is given by

$$
g\left(x_{1}, x_{2}\right)=h^{2}\left(x_{1}, x_{2}\right)\left(d x_{1} \otimes d x_{1}+d x_{2} \otimes d x_{2}\right)
$$

for some function $h$. In these coordinates the symplectic (area) form is given by

$$
w=h^{2}\left(x_{1}, x_{2}\right) d x_{1} \wedge d x_{2} .
$$

Introduce complex coordinates $(\xi, \bar{\xi})$ on the plane $\left(x_{1}, x_{2}\right)$ by

$$
\begin{aligned}
& \xi=\frac{1}{\sqrt{2}}\left(x_{1}+i x_{2}\right), \\
& \bar{\xi}=\frac{1}{\sqrt{2}}\left(x_{1}-i x_{2}\right) .
\end{aligned}
$$

Using $(\xi, \bar{\xi})$ we obtain

$$
g(\xi, \bar{\xi})=h^{2}(\xi, \bar{\xi})(d \xi \otimes d \xi+d \bar{\xi} \otimes d \bar{\xi})
$$

and

$$
w=-i h^{2}(\xi, \bar{\xi}) d \xi \wedge d \bar{\xi} .
$$

Hamilton's equations (3) become

$$
h^{2}(\xi, \bar{\xi}) d \xi \wedge d \bar{\xi}\left(X_{H}, \cdot\right)=d(i H) .
$$

For convenience we think of $h^{2}(\xi, \bar{\xi}) d \xi \wedge d \bar{\xi}$ as the new area form and $i H$ as the new hamiltonian function. Writing $X_{H}=\dot{\xi} \frac{\partial}{\partial \xi}+\dot{\bar{\xi}} \frac{\partial}{\partial \xi}$ the equations become

$$
h^{2}(\xi, \bar{\xi}) \dot{\bar{\xi}}=-i \frac{\partial H}{\partial \xi}
$$


We now show that Hally's equations can be written as a hamiltonian system on the conformal plane. The equations are

$$
\bar{\xi}_{n}^{\prime}=h^{-2}\left(\xi_{n}, \bar{\xi}_{n}\right)\left(\sum_{k}^{\prime}-i \frac{\Gamma_{k}}{\xi_{n}-\xi_{k}}+i \Gamma_{n} \frac{\partial}{\partial \xi_{n}} \log \left(h\left(\xi_{n}, \bar{\xi}_{n}\right)\right)\right) .
$$

Multiplying each term of the sum in the right hand side by $\frac{\bar{\xi}_{n}-\bar{\xi}_{k}}{\bar{\xi}_{n}-\bar{\xi}_{k}}$ and adding the zero $i \Gamma_{n} \frac{\partial}{\partial \xi_{n}} \log \left(h\left(\xi_{k}, \bar{\xi}_{k}\right)\right)$ to the right-hand side we obtain

$$
\begin{aligned}
\bar{\xi}_{n}^{\prime}=h^{-2}\left(\xi_{n}, \bar{\xi}_{n}\right)\left(\sum_{k}{ }^{\prime}-i \Gamma_{k} \frac{\partial}{\partial \xi_{n}} \log \left|\xi_{n}-\xi_{k}\right|^{2}+\right. & \\
& \left.i \Gamma_{n} \frac{\partial}{\partial \xi_{n}} \log \left(h\left(\xi_{n}, \bar{\xi}_{n}\right) h\left(\xi_{k}, \bar{\xi}_{k}\right)\right)\right) .
\end{aligned}
$$

Assuming that the sum of the vorticities is equal to zero we have

$$
\begin{aligned}
h^{2}\left(\xi_{n}, \bar{\xi}_{n}\right) \bar{\xi}_{n}^{\prime}=-i\left(\sum_{\xi}{ }^{\prime} \Gamma_{k} \frac{\partial}{\partial \xi_{n}} \log \left|\xi_{n}-\xi_{k}\right|^{2}+\right. & \\
& \left.\sum_{k}^{\prime} \Gamma_{k} \frac{\partial}{\partial \xi_{n}} \log \left(h\left(\xi_{n}, \bar{\xi}_{n}\right) h\left(\xi_{k}, \bar{\xi}_{k}\right)\right)\right),
\end{aligned}
$$

that is

$$
h^{2}\left(\xi_{n}, \bar{\xi}_{n}\right) \bar{\xi}_{n}^{\prime}=-i \sum_{k}^{\prime} \Gamma_{k} \frac{\partial}{\partial \xi_{n}} \log \left(h\left(\xi_{n}, \bar{\xi}_{n}\right) h\left(\xi_{k}, \bar{\xi}_{k}\right)\left|\xi_{n}-\xi_{k}\right|^{2}\right) .
$$

But those are Hamilton's equations

$$
w\left(X_{H}, \cdot\right)=d(i H)
$$

for the symplectic two-form

$$
\tilde{w}=\sum_{i=1}^{N} \Gamma_{i} h^{2}\left(\xi_{i}, \bar{\xi}_{i}\right) d \xi_{i} \wedge d \bar{\xi}_{i}
$$

and Hamiltonian function

$$
H=\frac{1}{2} \sum_{k, n}^{\prime} \Gamma_{k} \Gamma_{n} \log \left(h\left(\xi_{n}, \bar{\xi}_{n}\right) h\left(\xi_{k}, \bar{\xi}_{k}\right)\left|\xi_{n}-\xi_{k}\right|^{2}\right) .
$$

2.2. From Hally's to Bogolmonov's equations. Denote by $S^{2} \subset \mathbb{R}^{3}$ the two-dimensional sphere

$$
S^{2}=\left\{(x, y, z) \in \mathbb{R}^{3} \mid x^{2}+y^{2}+z^{2}=R^{2}\right\} .
$$

$S^{2}$ is a Riemannian manifold with the metric induced by the Euclidean metric in $\mathbb{R}^{3}$. Introduce spherical coordinates on $S^{2}$ trough the relations

$$
\begin{aligned}
& x=R \cos (\phi) \sin (\theta), \\
& y=R \sin (\phi) \sin (\theta), \\
& z=R \cos (\theta),
\end{aligned}
$$


with $0 \leq \phi<2 \pi, 0<\theta<\pi$.

Consider the conformal map $\sigma: S^{2} \rightarrow \mathbb{C}, \sigma(\theta, \phi)=u+i v$ where

$$
\begin{aligned}
& u=\tan (\theta / 2) \cos (\phi), \\
& v=\tan (\theta / 2) \sin (\phi) .
\end{aligned}
$$

The conformal factor is given by

$$
h(\xi, \bar{\xi})=\frac{2 R}{1+\xi \bar{\xi}}
$$

where $\xi=u+i v$. Now let $\vec{r}_{1}, \vec{r}_{2} \in S^{2} \subset \mathbb{R}^{3}$. The Euclidean distance $\left\|\vec{r}_{1}-\vec{r}_{2}\right\|^{2}$ in $\mathbb{R}^{3}$ can be computed in the conformal variables. In fact

$$
\begin{aligned}
\left\|\vec{r}_{1}-\vec{r}_{2}\right\|^{2} & =\left(x_{1}-x_{2}\right)^{2}+\left(y_{1}-y_{2}\right)^{2}+\left(z_{1}-z_{2}\right)^{2}, \\
& =R^{2}\left\{2-2 \cos \left(\theta_{1}\right) \cos \left(\theta_{2}\right)-2 \sin \left(\theta_{1}\right) \sin \left(\theta_{2}\right) \cos \left(\phi_{1}-\phi_{2}\right)\right\}, \\
& =4 \cos \left(\theta_{1}\right)^{2} \cos \left(\theta_{2}\right)^{2} R^{2}\left\{\left(u_{1}^{2}+v_{1}^{2}\right)+\left(u_{1}^{2}+v_{1}^{2}\right)-2 u_{1} v_{1}-2 u_{2} v_{2}\right\} \\
& =\frac{2 R}{1+\xi_{1} \vec{\xi}_{1}} \frac{2 R}{1+\xi_{2} \bar{\xi}_{2}}\left|\xi_{1}-\xi_{2}\right|^{2}
\end{aligned}
$$

that is

$$
\left\|\vec{r}_{1}-\vec{r}_{2}\right\|^{2}=h\left(\xi_{1}, \bar{\xi}_{1}\right) h\left(\xi_{2}, \bar{\xi}_{2}\right)\left|\xi_{1}-\xi_{2}\right|^{2} .
$$

Now consider the map $F: S^{2} \times \cdots \times S^{2} \rightarrow \mathbb{C}^{N}$ given by

$$
F\left(\vec{r}_{1}, \vec{r}_{2}, \ldots, \vec{r}_{N}\right)=\left(\sigma\left(\vec{r}_{1}\right), \sigma\left(\vec{r}_{2}\right), \ldots, \sigma\left(\vec{r}_{N}\right)\right) .
$$

The pull back by $F$ of the symplectic form

$$
w=\sum_{i=1}^{N} \Gamma_{i} h^{2}\left(\xi_{i}, \bar{\xi}_{i}\right) d \xi_{i} \wedge d \bar{\xi}_{i}
$$

is just

$$
F^{*} \tilde{w}=\sum_{i=1}^{N} \Gamma_{i} w_{i}
$$

where by $w_{i}$ we denote the standard area form

$$
w_{i}=x_{i} d y_{i} \wedge d z_{i}+y_{i} d z_{i} \wedge d x_{i}+z_{i} d x_{i} \wedge d y_{i}
$$

induced by the Euclidean metric on $S_{i}^{2}$. The pull back of the Hamiltonian function

$$
H=\frac{1}{2} \sum_{k, n}^{\prime} \Gamma_{k} \Gamma_{n} \log \left(h\left(\xi_{n}, \bar{\xi}_{n}\right) h\left(\xi_{k}, \bar{\xi}_{k}\right)\left\|\xi_{n}-\xi_{k}\right\|^{2}\right)
$$

is

$$
F^{*} H=\frac{1}{2} \sum_{k, n}^{\prime} \Gamma_{k} \Gamma_{n} \log \left(\left\|\vec{r}_{k}-\vec{r}_{n}\right\|^{2}\right) .
$$


Hamilton's equations for Hamiltonian function (12) and symplectic form (11) are precisely Bogolmonov's equations for $N$ interacting vortices on a sphere.

We notice that Hally's equations are equivalent to Bogolmonov's equations only for the case where all the vorticities sums up to zero. Therefore, in dealing with the general vorticity case, one must use Bogomolnov's equations.

\section{Equations on the Symmetric Ellipsoid - Perturbation APPROACH}

Let $E^{2} \subset \mathbb{R}^{3}$ represent the two dimensional ellipsoid of revolution

$$
\frac{x^{2}}{R^{2}}+\frac{y^{2}}{R^{2}}+\frac{z^{2}}{R^{2}(1+\epsilon)}=1 \text {. }
$$

Where $R>0$ and $|\epsilon|<<1$. If $\epsilon>0$ the ellipsoid is prolate. In this case the eccentricity $e$ of the generating ellipse is given by $e=\sqrt{\frac{\epsilon}{(1+\epsilon)}}$. If $\epsilon<0$ the ellipsoid is oblate and the eccentricity of the generating ellipse is given by $e=\sqrt{-\epsilon}$. We observe that $\epsilon=\mathcal{O}\left(e^{2}\right)$ and therefore the truncated first order system is expected to approximate the real system for quite high values of the eccentricity.

We introduce coordinates on $E^{2}$ trough the relations

$$
\begin{aligned}
& x=R \cos (\phi) \sin (\theta), \\
& y=R \sin (\phi) \sin (\theta), \\
& z=R \sqrt{1+\epsilon} \cos (\theta),
\end{aligned}
$$

with $0 \leq \phi<2 \pi, 0<\theta<\pi$. If $\epsilon=0$ the surface is a sphere of radius $R$. In this case the projection

$$
\begin{aligned}
& u=\tan (\theta / 2) \cos (\phi), \\
& v=\tan (\theta / 2) \sin (\phi)
\end{aligned}
$$

is a conformal transformation. We want to find a conformal transformation from $E^{2}$ to the conformal plane when $\epsilon \neq 0$. By the symmetry of the ellipsoid the conformal factor must depend only on the radial variable $r$ and not on the angular variable $\psi$. We look for a transformation of the form

$$
\begin{aligned}
& \theta=2 \arctan (r)+2 \sum_{i=1}^{\infty} f_{i}(r) \epsilon^{i}, \\
& \phi=\psi .
\end{aligned}
$$

We observe that when $\epsilon=0$ the inverse of the above transformation is just transformation (15) written in polar coordinates.

The symmetry of the ellipsoid implies that the equator is a circle of radius $R$. In the $(\theta, \phi)$ coordinates the equator has parametrization given by $\theta=\frac{\pi}{2}$ and $\phi \in[0,2 \pi)$. Therefore in the conformal coordinates $(u, v)$ the equator becomes the circle of radius $r=u^{2}+v^{2}=1$. We remark that the conformal 
factor for the ellipsoid, when restricted to the circle of radius 1 is just the usual one for the sphere since the ellipsoid $E^{2}$ and the $S^{2}$ coincide at the equator. This is of utmost importance for what follows. In fact, since we will impose conformality at each order this trivial observation implies that $f_{i}(1)=0$ for all $i$. This is the condition that will guarantee the uniqueness of our series expansion.

Remark 3.1. Alternatively (and equivalently), the uniqueness of the expansion can be obtained by imposing that the symmetry condition $h(r)=$ $\frac{1}{r} h\left(r^{-1}\right)$ has to be satisfied at all orders.

The algorithmic determination of the $f_{i}(r)$ is obtained by imposing conformality at each order and follows the standard procedures of classical perturbation theory: Truncate the series at order $n$, find the conditions to be satisfied, and them assume those conditions to find the term of order $n+1$. To find $f_{1}(r)$ we impose that

$$
\begin{aligned}
& \theta=2 \arctan (r)+2 f_{1}(r) \epsilon, \\
& \phi=\psi .
\end{aligned}
$$

generates a conformal transformation up to first order in $\epsilon$.

The line element for the ellipsoid is

$$
d s^{2}=R^{2} \sin ^{2}(\theta) d \phi^{2}+R^{2}\left(1+\epsilon \sin ^{2}(\theta)\right) d \theta^{2} .
$$

We want to write $d s^{2}$ in terms of $r$ and $\phi$. To this goal it suffices writing $\sin (\theta)$ and $d \theta$ in terms of $r$. But

$$
\begin{aligned}
& \sin (\theta)=\sin \left(2 \arctan (r)+2 f_{1}(r) \epsilon\right), \\
& =\sin (2 \arctan (r))+2 \cos (2 \arctan (r)) f_{1}(r) \epsilon+\mathcal{O}\left(\epsilon^{2}\right) \\
& =2 \sin (\arctan (r)) \cos (\arctan (r))+ \\
& +2\left(\cos (\arctan (r))^{2}-\sin (\arctan (r))^{2}\right) f_{1}(r) \epsilon+\mathcal{O}\left(\epsilon^{2}\right), \\
& =2 \frac{r}{1+r^{2}}+2 \frac{\left(1-r^{2}\right)}{1+r^{2}} f_{1}(r) \epsilon+\mathcal{O}\left(\epsilon^{2}\right) .
\end{aligned}
$$

This gives

$$
\sin (\theta)^{2}=\frac{4 r^{2}}{\left(1+r^{2}\right)^{2}}+\frac{8 r\left(1-r^{2}\right)}{\left(1+r^{2}\right)^{2}} f_{1}(r) \epsilon+\mathcal{O}\left(\epsilon^{2}\right) .
$$

Also

Therefore

$$
d \theta=\left(2 \frac{1}{1+r^{2}}+2 \frac{\partial f_{1}(r)}{\partial r} \epsilon+\mathcal{O}\left(\epsilon^{2}\right)\right) d r
$$

$$
d \theta^{2}=\left(4 \frac{1}{\left(1+r^{2}\right)^{2}}+\frac{8}{1+r^{2}} \frac{\partial f_{1}(r)}{\partial r} \epsilon+\mathcal{O}\left(\epsilon^{2}\right)\right) d r^{2} .
$$


Finally

$$
\begin{aligned}
& d s^{2}=\frac{4 R^{2}}{\left(1+r^{2}\right)^{2}}\left\{\left[1+\frac{2\left(1-r^{2}\right)}{r} f_{1}(r) \epsilon+\mathcal{O}\left(\epsilon^{2}\right)\right] r^{2} d \psi^{2}+\right. \\
& \left.+\left[1+\left(\frac{4 r^{2}}{\left(1+r^{2}\right)^{2}}+2\left(1+r^{2}\right) \frac{\partial f_{1}(r)}{\partial r}\right) \epsilon+\mathcal{O}\left(\epsilon^{2}\right)\right] d r^{2}\right\} .
\end{aligned}
$$

This will be conformal to the plane if it is a multiple of $r^{2} d \psi^{2}+d r^{2}$. We observe that if $\epsilon=0$ then the transformation is conformal with conformal factor $\sqrt{\frac{4 R^{2}}{\left(1+r^{2}\right)^{2}}}$. For $\epsilon \neq 0$ the only way this transformation can be conformal for all values of $\epsilon$ is if the coefficient of $\epsilon$ in the first square bracket is equal to the coefficient of $\epsilon$ in the second square bracket, that is if

$$
\left(1+r^{2}\right) \frac{\partial f_{1}(r)}{\partial r}-\frac{\left(1-r^{2}\right)}{r} f_{1}(r)=-\frac{2 r^{2}}{\left(1+r^{2}\right)^{2}} .
$$

This is a linear differential equation for $f_{1}(r)$. Its general solution (for $r>0$ ) is equal to

$$
f_{1}(r)=-\frac{\left(\frac{1}{1+r^{2}}+c\right)}{\left(1+r^{2}\right)} r .
$$

Now by remark (3.1) we must have $f_{1}(1)=0$ what implies $c=-\frac{1}{2}$. Finally we have

$$
f_{1}(r)=\frac{1-r^{2}}{\left(1+r^{2}\right)^{2}} r
$$

Transformation (16) becomes

$$
\begin{aligned}
& \theta=2 \arctan (r)+\frac{\left(1-r^{2}\right)}{\left(1+r^{2}\right)^{2}} r \epsilon+\sum_{i=2}^{\infty} f_{i}(r) \epsilon^{i}, \\
& \phi=\psi .
\end{aligned}
$$

The conformal factor is, to first order given by,

$$
h(r)^{2}=\frac{4 R^{2}}{\left(1+r^{2}\right)^{2}}\left\{1+2 \frac{\left(1-r^{2}\right)^{2}}{\left(1+r^{2}\right)^{2}} \epsilon\right\}
$$

that is

$$
h(r)=\frac{2 R}{\left(1+r^{2}\right)}\left\{1+\frac{\left(1-r^{2}\right)^{2}}{\left(1+r^{2}\right)^{2}} \epsilon\right\}
$$

We write

$$
h(r)=h_{0}(r)+h_{1}(r) \epsilon+\mathcal{O}\left(\epsilon^{2}\right),
$$

where

$$
h_{0}(r)=\frac{2 R}{\left(1+r^{2}\right)} \text { and } h_{1}(r)=2 R \frac{\left(1-r^{2}\right)^{2}}{\left(1+r^{2}\right)^{3}} .
$$

For future reference we observe that in terms of the variables $\theta$ and $\phi$ we obtain

$$
h_{0}(r)=2 R \cos ^{2}(\theta / 2) \text { and } h_{1}(r)=4 R \cos (\theta) \cos ^{2}(\theta / 2) .
$$


Remark 3.2. The above expansion can not be carried explicitly further. In fact, the equation for the second order factor will be

$$
\frac{d f_{2}}{d r}+\frac{1}{2} \frac{r\left(r^{2}-1\right)}{r^{2}+1} f_{2}+\frac{1-13 r^{2}+46 r^{4}-r^{10}-26 r^{6}+9 r^{8}}{4\left(1+r^{2}\right)^{5}}=0 .
$$

The general solution for this equation is given by

$$
f_{2}(r)=\frac{\sqrt{1+r^{2}}}{4 e^{r^{2} / 4}}\left(\int \frac{\left(\left(r^{2}-1\right)^{2}+4 r^{2}\right)\left(r^{6}-3 r^{4}+7 r^{2}-1\right)}{\left(1+r^{2}\right)^{11 / 2}} e^{1 / 4 r^{2}} d r+4 c\right)
$$

where $c$ is an arbitrary constant. The above integral can not be computed using elementary functions.

3.1. N-Vortex Equations: Using the conformal factor just found we will write the N-vortex equations on the ellipsoid of revolution up to first order terms in $\epsilon$. We use the coordinates $(\theta, \phi)$ introduced trough equations (14). The symplectic two-form becomes

$$
w=\sqrt{1+\epsilon} d \phi \wedge d(\cos (\theta)) .
$$

Observing that

$$
\sqrt{1+\epsilon} d \phi \wedge d(\cos (\theta))\left(X_{H}, \cdot\right)=d \phi \wedge d(\cos (\theta))\left(\sqrt{1+\epsilon} X_{H}, \cdot\right),
$$

we define the vector field $\tilde{X}=\sqrt{1+\epsilon} X_{H} . \tilde{X}$ satisfies Hamilton's equations

$$
d \phi \wedge d(\cos (\theta))(\tilde{X}, \cdot)=d H
$$

and the flow $\phi_{s}(x)$ of the vector field $\tilde{X}_{h}$ is a time reparametrization of the flow $\phi_{t}(x)$ induced by $X_{H}$. In fact $t=\sqrt{1+\epsilon} s$. We therefore consider equation (20) with $H$ and $h$ given by (9) and (19) respectively . $H$ is given by

$H=\frac{1}{2} \sum_{i, j}^{\prime} \Gamma_{i} \Gamma_{j} \ln \left[\left(h_{0}\left(r_{i}\right)+\epsilon h_{1}\left(r_{i}\right)\right)\left(h_{0}\left(r_{j}\right)+\epsilon h_{1}\left(r_{j}\right)\right)\left|\xi_{i}-\xi_{j}\right|^{2}+\mathcal{O}\left(\epsilon^{2}\right)\right]$

that is

$H=\frac{1}{2} \sum_{i, j}^{\prime} \Gamma_{i} \Gamma_{j}\left[\ln \left(h_{0}\left(r_{i}\right) h_{0}\left(r_{j}\right)\left|\xi_{i}-\xi_{j}\right|^{2}\right)+\epsilon\left(\frac{h_{1}\left(r_{i}\right)}{h_{0}\left(r_{i}\right)}+\frac{h_{1}\left(r_{j}\right)}{h_{0}\left(r_{j}\right)}\right)\right]+\mathcal{O}\left(\epsilon^{2}\right)$

The Hamiltonian function $H$ is a function on the variables $(\theta, \phi)$. Moreover, the symplectic two-form (20) is the standard symplectic form over the sphere. Therefore, we write

$$
\begin{array}{r}
H=\frac{1}{2} \sum_{i, j}{ }^{\prime} \Gamma_{i} \Gamma_{j}\left[\ln \left(2-2 \cos \left(\theta_{i}\right) \cos \left(\theta_{j}\right)-2 \sin \left(\theta_{i}\right) \sin \left(\theta_{j}\right) \cos \left(\phi_{i}-\phi_{j}\right)\right)\right. \\
\left.+\epsilon\left(\cos \left(\theta_{i}\right)^{2}+\cos \left(\theta_{j}\right)^{2}\right)\right]+\mathcal{O}\left(\epsilon^{2}\right) .
\end{array}
$$


We conclude that, to first order in $\epsilon$ the dynamics of the $\mathrm{N}$ vortices on the ellipsoid is determined by the hamiltonian system with Hamiltonian function $H=\frac{1}{2} \sum_{i, j}^{\prime} \Gamma_{i} \Gamma_{j}\left[\ln \left(2-2 \cos \left(\theta_{i}\right) \cos \left(\theta_{j}\right)-2 \sin \left(\theta_{i}\right) \sin \left(\theta_{j}\right) \cos \left(\phi_{i}-\phi_{j}\right)\right)\right.$

$$
\left.+\epsilon\left(\cos \left(\theta_{i}\right)^{2}+\cos \left(\theta_{j}\right)^{2}\right)\right]
$$

and symplectic two-form

$$
w=\sum_{i=1}^{N} \Gamma_{i} d \phi_{i} \wedge d\left(\cos \left(\theta_{i}\right)\right) .
$$

Alternatively, in cartesian coordinates the above Hamiltonian system becomes

$$
H=\frac{1}{2} \sum_{i, j}^{\prime} \Gamma_{i} \Gamma_{j} \log \left\|\vec{r}_{i}-\vec{r}_{j}\right\|^{2}+\frac{\epsilon}{2} \sum_{i, j}^{\prime} \Gamma_{i} \Gamma_{j}\left(z_{i}^{2}+z_{j}^{2}\right),
$$

with symplectic two-form given by

$$
w=\sum_{i=1}^{n} \Gamma_{i}\left(x_{i} d y_{i} \wedge d z_{i}+y_{i} d z_{i} \wedge d x_{i}+z_{i} d x_{i} \wedge d y_{i}\right) .
$$

4. Applichtions: The $\mathrm{N}=2,3$ Cases.

4.1. Two-Vortex problem. When $N=2$ the hamiltonian system given by (21) and (22) reduces to

$$
\begin{aligned}
& \dot{\vec{r}}_{1}=\Gamma_{2} \frac{\vec{r}_{2} \times \vec{r}_{1}}{\left\|\vec{r}_{1}-\vec{r}_{2}\right\|^{2}}+\epsilon \Gamma_{2} \vec{r}_{1} \times z_{1} \hat{z}, \\
& \dot{\vec{r}}_{2}=\Gamma_{1} \frac{\vec{r}_{1} \times \vec{r}_{2}}{\left\|\vec{r}_{1}-\vec{r}_{2}\right\|^{2}}+\epsilon \Gamma_{1} \vec{r}_{2} \times z_{2} \hat{z} .
\end{aligned}
$$

Doing the following change of variables

$$
\begin{aligned}
& \vec{w}=\Gamma_{1} \overrightarrow{r_{1}}-\Gamma_{2} \overrightarrow{r_{2}}, \\
& \vec{c}=\Gamma_{1} \overrightarrow{r_{1}}+\Gamma_{2} \overrightarrow{r_{2}} .
\end{aligned}
$$

we obtain

$$
\begin{aligned}
& \dot{\vec{w}}=\frac{\vec{c} \times \vec{w}}{\left\|\alpha_{1} \vec{c}-\alpha_{2} \vec{w}\right\|^{2}}+\epsilon\left(\Delta_{1}\left(c_{z}, w_{z}\right) \vec{c} \times \hat{z}+\Delta_{2}\left(c_{z}, w_{z}\right) \vec{w} \times \hat{z}\right) \\
& \dot{\vec{c}}=\epsilon\left(\Delta_{2}\left(c_{z}, w_{z}\right) \vec{c} \times \hat{z}+\Delta_{1}\left(c_{z}, w_{z}\right) \vec{w} \times \hat{z}\right) ;
\end{aligned}
$$

where

$$
\begin{gathered}
\alpha_{1} \equiv \frac{1}{2} \frac{\Gamma_{2}-\Gamma_{1}}{\Gamma_{1} \Gamma_{2}} \quad, \quad \alpha_{2} \equiv \frac{1}{2} \frac{\Gamma_{1}+\Gamma_{2}}{\Gamma_{1} \Gamma_{2}} \\
\Delta_{1} \equiv \frac{c_{z}+w_{z}}{4 \Gamma_{1}^{2}}+\frac{c_{z}-w_{z}}{4 \Gamma_{2}^{2}} \quad \text { and } \quad \Delta_{2} \equiv \frac{c_{z}+w_{z}}{4 \Gamma_{1}^{2}}-\frac{c_{z}-w_{z}}{4 \Gamma_{2}^{2}} .
\end{gathered}
$$

Equations (25) are singular when $\alpha_{1} \vec{c}=\alpha_{2} \vec{w}$, i.e. $\vec{r}_{1}=\vec{r}_{2}$. When $\epsilon=0$ equations (25) reduces to a linear system and can be easily solved. Those are the well known solutions for the two-vortex problem over the sphere: In 
a system of coordinates such that its z-axis is in the same direction as the center of vorticity $\vec{c}$ the solutions are given in the $\vec{r}_{1}, \vec{r}_{2}$ variables by

$$
\begin{aligned}
& \overrightarrow{r_{1}}(t)=\frac{1}{2 \Gamma_{1}}\left(\mu \cos (\theta), \mu \sin (\theta),\|\vec{c}\|+w_{z}\right), \\
& \overrightarrow{r_{2}}(t)=\frac{1}{2 \Gamma_{2}}\left(-\mu \cos (\theta),-\mu \sin (\theta),\|\vec{c}\|-w_{z}\right)
\end{aligned}
$$

where

$$
\theta \equiv \gamma t+\phi
$$

with $\phi$ a constant and

$$
\gamma=\frac{\|\vec{c}\|}{\alpha_{2}^{2} \mu^{2}+\left(\alpha_{2} w_{z}-\alpha_{1}\|\vec{c}\|\right)^{2}},
$$

with

$$
\mu^{2}=w_{x}^{2}+w_{y}^{2}=w^{2}-w_{z}^{2} \text { and } \mu \geq 0 .
$$

We observe that all solutions are periodic and the relative distance between the vortices is a first integral.

When $\epsilon \neq 0$ the center of vorticity vector $\vec{c}$ is not preserved . In fact, only it's $z$ component $c_{z}$ is preserved. This first integral implies, by dimensionality that system is integrable. Since $\left\|\vec{r}_{1}\right\|=\left\|\vec{r}_{2}\right\|=1$ we obtain that

$$
\|\vec{c}\|^{2}+\|\vec{w}\|^{2}=k_{1} \text { and } \vec{c} \cdot \vec{w}=k_{2}
$$

are constants of motion. Despite being integrable system (25) seems not to admit explicit solutions for a general initial condition. Their relative equilibria can be easily characterized in the cases $\left|\Gamma_{1}\right|=\left|\Gamma_{2}\right| \equiv \Gamma$. Then we obtain that

$$
\|\vec{c}\|^{2}+\|\vec{w}\|^{2}=\Gamma \text { and } \vec{c} \cdot \vec{w}=0 .
$$

First we characterize the solutions such that $\left\|\vec{r}_{1}(t)-\vec{r}_{2}(t)\right\|$ is constant along the motion. Since

$$
\left\|\vec{r}_{1}-\vec{r}_{2}\right\|^{2}=\|\vec{c}\|^{2} \alpha_{1}^{2}+\|\vec{w}\|^{2} \alpha_{2}^{2}
$$

and since for $\left|\Gamma_{1}\right|=\left|\Gamma_{2}\right|$ either $\alpha_{1}=0$ or $\alpha_{2}=0$ it follows that $\|\vec{c}\|^{2}$ and $\|\vec{w}\|^{2}$ are constants for the relative equilibria. Therefore

$$
\vec{c} \cdot \dot{\vec{c}}=\epsilon \Delta_{2} \vec{c} \cdot(\vec{w} \times \hat{z})=0 .
$$

$\Delta_{2}=0$ implies that $w_{z}$ is a constant and therefore $\dot{w}_{z}=0$. From the equations it follows that $\vec{c} \cdot(\vec{w} \times \hat{z})=0$. This implies that the only way (28) can be zero is if $\vec{c} \cdot(\vec{w} \times \hat{z})=0$. We arrive at the result that in a relative equilibria $\|\vec{c}\|^{2},\|\vec{w}\|^{2}$ and $w_{z}$ are constants and that the vectors $\vec{c}, \vec{w}$ and $\hat{z}$ are always in the same plane. The vectors $\vec{c}$ and $\vec{w}$ will precess around the $z$-axis with constant angular velocity and keeping their relative orientation. It follows from the definition that $\vec{r}_{1}$ and $\vec{r}_{2}$ will also precess around the $z$-axis with constant angular velocity and keeping their relative orientation. Therefore the only solutions for which $\left\|\vec{r}_{1}(t)-\vec{r}_{2}(t)\right\|$ are constants are precisely the relative equilibria. 
We compute the angular velocity of the relative equilibria in the cases where $\left|\Gamma_{1}\right|=\left|\Gamma_{2}\right|$.

Case $\Gamma_{1}=\Gamma_{2}$ : If $\Gamma_{1}=\Gamma_{2}=\Gamma$ then $\alpha_{1}=0$ and $\alpha_{2}=\Gamma^{-1}$. Assuming that $c_{x}=A \cos (\Omega t), c_{y}=A \sin (\Omega t), w_{x}=B \cos (\Omega t)$ and $w_{y}=B \sin (\Omega t)$ we obtain that the angular velocity is given by

$$
\Omega=\frac{\Gamma^{2}}{\|\vec{w}\|^{2}}\left(c_{z}-\frac{\sqrt{\|\vec{c}\|^{2}-c_{z}^{2}}}{\mu}\right)+\frac{\epsilon}{2 \Gamma^{2}}\left(c_{z}+w_{z} \frac{\sqrt{\|\vec{c}\|^{2}-c_{z}^{2}}}{\mu}\right) .
$$

We observe that the perturbation factor in the angular velocity is proportional to $\frac{1}{\Gamma^{2}}$ and therefore the deviation from the corresponding spherical relative equilibria should be bigger for smaller vorticities. We also observe that when $\epsilon=0$ then, taking as before $\|\vec{c}\|=\left|c_{z}\right|$, the above expression reduces to (27).

Case $\Gamma_{1}=-\Gamma_{2}$ : If $\Gamma_{1}=-\Gamma_{2}=\Gamma$ then $\alpha_{1}=\Gamma^{-1}$ and $\alpha_{2}=0$. Proceeding as before we obtain

$$
\Omega=\frac{\Gamma^{2}}{\|\vec{c}\|^{2}}\left(c_{z}-\frac{\sqrt{\|\vec{c}\|^{2}-c_{z}^{2}}}{\mu}\right)+\frac{\epsilon}{2 \Gamma^{2}}\left(c_{z}+w_{z} \frac{\sqrt{\|\vec{c}\|^{2}-c_{z}^{2}}}{\mu}\right) .
$$

The perturbation term is equal in both cases and again, as $\epsilon \rightarrow 0$ we will have $w \rightarrow \gamma$.

4.2. The 3-Vortex problem. The symmetry breaking when $\epsilon \neq 0$ is expected to destroy the integrability of the three-vortices spherical problem. In fact, when $\epsilon=0$ the center of vorticity vector $\vec{c}=\Gamma_{1} \vec{r}_{1}+\Gamma_{2} \vec{r}_{2}+\Gamma_{3} \vec{r}_{3}$ is conserved. The integrability follows by dimensionality. When $\epsilon \neq 0$, only it's z-component $c_{z}=\Gamma_{1} z_{1}+\Gamma_{2} z_{2}+\Gamma_{3} z_{3}$ is conserved. That the integrability is destroyed is shown numerically in this section. We do not pursue a complete numerical study of the problem. This will addressed in a future work.

To calculate the Poincaré sections for the three-vortex problem we will introduce an appropriate coordinate system. First we introduce a cylindrical system of coordinates $\left(z_{1}, z_{2}, z_{3}, \phi_{1}, \phi_{2}, \phi_{3}\right)$ with $\phi_{i} \in[0,2 \pi)$ and $z_{i} \in(-1,1)$ for $i=1,2$. The transformation is defined trough

$$
\begin{aligned}
& x_{i}=\sqrt{1-z_{i}^{2}} \cos \left(\phi_{i}\right), \\
& y_{i}=\sqrt{1-z_{i}^{2}} \sin \left(\phi_{i}\right), \\
& z_{i}=z_{i} .
\end{aligned}
$$

Since

$$
w=\sum_{i=1}^{3} \Gamma_{i} x_{i} d y_{i} \wedge d z_{i}+y_{i} d z_{i} \wedge d x_{i}+z_{i} d x_{i} \wedge d y_{i}=\sum_{i=1}^{3} \Gamma_{i} d \phi_{i} \wedge d z_{i},
$$


this is a symplectic transformation and the equations in cylindrical coordinates are given by

$$
\dot{\phi}_{i}=\frac{1}{\Gamma_{i}} \frac{\partial H}{\partial z_{i}} \text { and } \dot{z}_{i}=-\frac{1}{\Gamma_{i}} \frac{\partial H}{\partial \phi_{i}} \text { for } i=1,2 .
$$

To reduce the symmetry we define the coordinates

$$
\begin{aligned}
& q_{1}=\Gamma_{1} z_{1}-\Gamma_{3} z_{3}, \\
& q_{2}=\Gamma_{1} z_{1}-\Gamma_{2} z_{2}, \\
& q_{3}=\Gamma_{1} z_{1}+\Gamma_{2} z_{2}+\Gamma_{3} z_{3}, \\
& p_{1}=\frac{\phi_{1}}{3}+\frac{\phi_{2}}{3}-2 \frac{\phi_{3}}{3} \\
& p_{2}=\frac{\phi_{1}}{3}-2 \frac{\phi_{2}}{3}+\frac{\phi_{3}}{3}, \\
& p_{3}=\frac{\phi_{1}}{3}+\frac{\phi_{2}}{3}+\frac{\phi_{3}}{3} .
\end{aligned}
$$

It follows that

$d q_{1} \wedge d p_{1}+d q_{2} \wedge d p_{2}+d q_{3} \wedge d p_{3}=\Gamma_{1} d z_{1} \wedge d \phi_{1}+\Gamma_{2} d z_{2} \wedge d \phi_{2}+\Gamma_{3} d z_{3} \wedge d \phi_{3}$.

Therefore the new system of coordinates forms a canonical system. We observe that $q_{3}(t)=c_{z}$ and therefore $\dot{q}_{3}(t)=\frac{\partial H}{\partial p_{3}}=0$. Then $p_{3}$ is a cyclic variable and the Hamiltonian (21) is given by

$$
H=H\left(q_{1}, q_{2}, p_{1}, p_{2}, c_{z}\right) .
$$

Since this Hamiltonian is now defined in a four dimensional phase-space, the Poincarè maps can be visualized. The equations are integrated numerically using the Fehlberg-7(8) Runge-Kuta. The relative error in energy is kept smaller then $10^{-10}$. A constant step size equals to $10^{-3}$ is used in all integrations.

Figure 1 show the pictures of three Poincarè maps. The onset of chaotic behavior is clear. It appears (as expected) around the homoclinic solution present in the integrable case $\epsilon=0.00$. We observe that for $\epsilon=0.05$ and $\epsilon=0.10$ the eccentricity of the generating ellipses are 0.22 and 0.33 respectively.

\section{REFERENCES}

[1] H. Helmholtz On integrals of the hydrodynamical equations wich express vortexmotion, Philos. Mag. 33(4) (1887)

[2] G. Kirchhoff Vorlensungen Uber Matematische Physik, 3rd ed. (Teubner, Leipzig, 1883)

[3] V. A. Bogolmonov Dynamics of vorticity at a sphere, Fluid. Dyn. (USSR) 6, 863-870 (1977)

[4] D. Hally Stability of streets of vortices on surfaces of revolution with a reflection symetry, J. Math. Phys.21 (1) 211-217 (1979) 
[5] Y. Kimura and H. Okamoto Vortex motion on a sphere, J. Phys. Soc. Japan 56 (12) 4203-4206 (1987)

[6] V. A. Bogolmonov Two dimensional fluid dynamics on a sphere, Izv. Atmos. Ocean. Phys. 15, 18-22 (1979)

[7] Newton, P. K.; The $N$-vortex problem. Applied Mathematical Sciences, 145. SpringerVerlag, New York, 2001.

[8] Kidambi, R.; Newton, P. K. Motion of three point vortices on a sphere. Phys. D 116 (1998), no. 1-2, 143-175.

[9] Marsden, J. E.; Ratiu, T. S. Introduction to mechanics and symmetry. A basic exposition of classical mechanical systems. Second edition. Texts in Applied Mathematics, 17. Springer-Verlag, New York, 1999. 

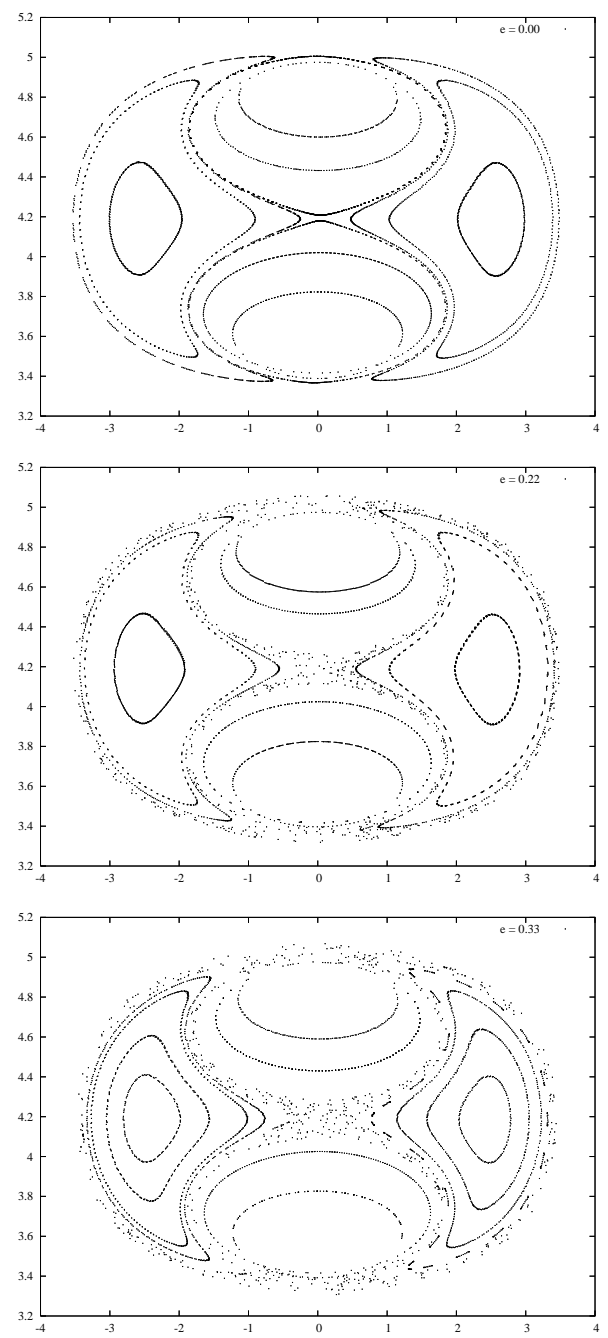

Figure 1: Poincarè maps for the Three-vortices problem. Here $\Gamma_{1}=\Gamma_{2}=\Gamma_{3}=$ 3 , Energy $=-\frac{1}{2}, c_{z}=-\frac{1}{4} . q_{2}$ is the horizontal axis and $p_{2}$ is the vertical axis. The values of $\epsilon$ are, from top to bottom, $\epsilon=0.00,0.05,0.10$ respectively. 